\title{
A rare neurologic deficiency in HaNDL syndrome: cranial neuropathy
}

Dilcan Kotan ${ }^{1}$, Saadet Sayan², Semra Alacam Koksal ${ }^{3}$

${ }^{1}$ Neurology Department, Ataturk University Medical Faculty, Erzurum, Turkey
${ }^{2}$ Department of Neurology, SB Sakarya University Research and Training Hospital,
Sakarya, Turkey
${ }^{3}$ Department of Neurology, Sakarya University Faculty of Medicine, Sakarya, Turkey

Submitted: 4 May 2016

Accepted: 7 June 2016

Arch Med Sci Atheroscler Dis 2016; 1: e49-e52

DOI: 10.5114/amsad.2016.60799

Copyright @ 2016 Termedia \& Banach

HaNDL syndrome is a neurologic disease characterized by headache, neurologic deficits and lymphocytic pleocytosis in the cerebrospinal fluid (CSF) $[1,2]$. Diagnostic criteria are: moderate or severe headache attacks lasting a few hours, transient neurologic symptoms accompanied by headaches or occurring after the headache, lymphocytes $>15$ cells $/ \mathrm{ml}$ in CSF (CSF culture is normal etiologic testing), and recurrent attacks of headaches and neurological symptoms within about 3 months [2]. It is associated with self-limited, monophasic progression, therapeutic interventions are unnecessary and resolves without sequelae within a few months [3]. Here, we describe the first case presenting with multiple cranial nerve palsy without increased intracranial pressure (ICP) which was diagnosed as HaNDL syndrome.

A 34-year-old woman presented with throbbing headache which spread to the right eye and head for 20 days, double vision side by side and facial numbness on the right side for 2.5 weeks. There was no history of systemic disease. Neurologic examination was normal except limited right lateral gaze and hypoesthesia in the right maxillary space of the trigeminal nerve (Figure 1). Fundoscopic examination was normal. Headache was resistant to the use of analgesics and triptans. Computed tomography (CT), diffusion weighted (DW) magnetic resonance (MRI), cranial MRI, cranial magnetic resonance venography (MRV), and cranial MRI with contrast enhancement were within normal limits (Figures 2 A-E). 100/ $\mathrm{mm}^{3}$ lymphocytes was observed in CSF at direct examination after lumbar puncture. Cerebrospinal fluid protein was $33.5(15-45 \mathrm{mg} / \mathrm{dl})$ and CSF pressure, cytological and biochemical were normal. There was no microorganism isolated in CSF culture. On electrophysiological examination, velocity and amplitude of motor and sensory nerve conduction and repetitive nerve stimulation were within normal limits. Carotid-vertebral Doppler ultrasonography was normal. Tests required for exclusion of infectious and vasculitic events, HSV type 1, type 2, CEA, CA 125, CA 15-3, CA 19-9, AFP, anti-ENA panel (RNP/Sm, SS-A, SS-B, Rover 52, Scl-70, PM-Scl, Jo-1, centromere B dsDNA, nucleosome, histone, ribosomal $P$ protein, AMA-M2), ASMA, and AMA were negative. Genetic examination of CADASIL, which was requested for the differential diagnosis due to the presence of a family history of migraine, was negative. She was suspected to have HaNDL syndrome and was administered symptomatic treatment, but right peripheral facial palsy and right abducens paralysis added to clinic symptoms followed for 15 days (Figures $3 \mathrm{~A}-\mathrm{C}$ ).

\author{
Corresponding author: \\ Dilcan Kotan \\ Neurology Department \\ Ataturk University \\ Medical Faculty \\ 25050 Erzurum, Turkey \\ Phone: 4882143030 \\ E-mail: dilcankotan@ \\ yahoo.com
}



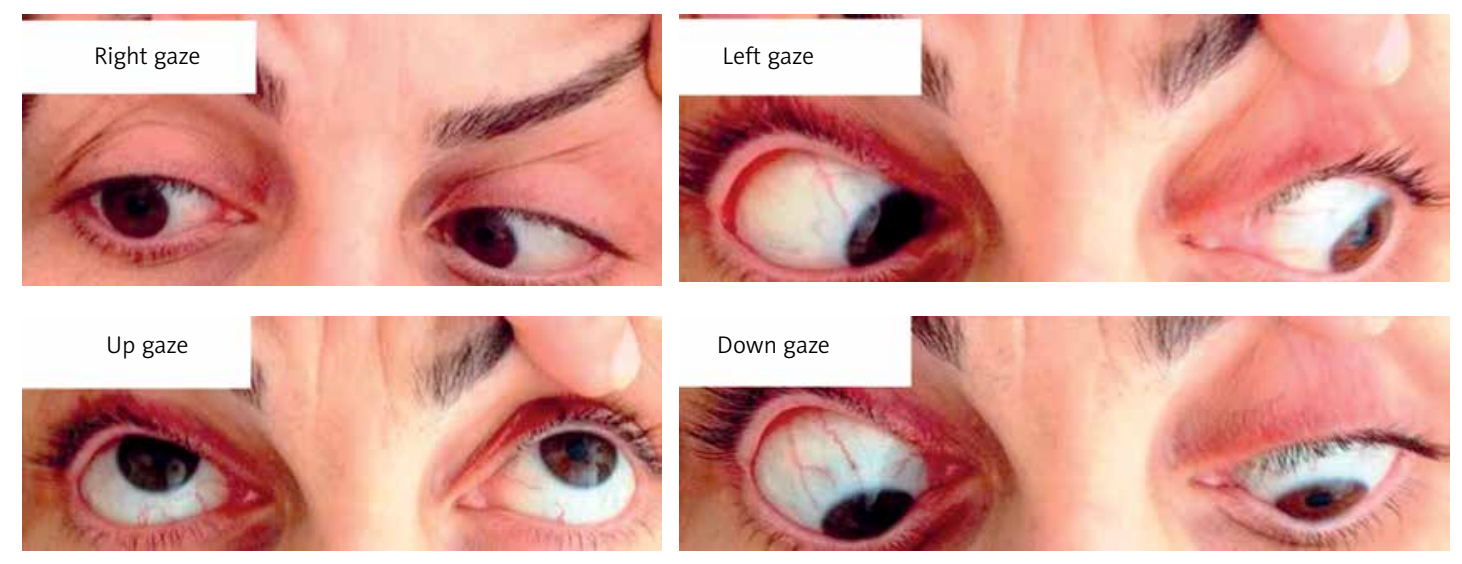

Figure 1. Limited right lateral gaze on neurologic examination
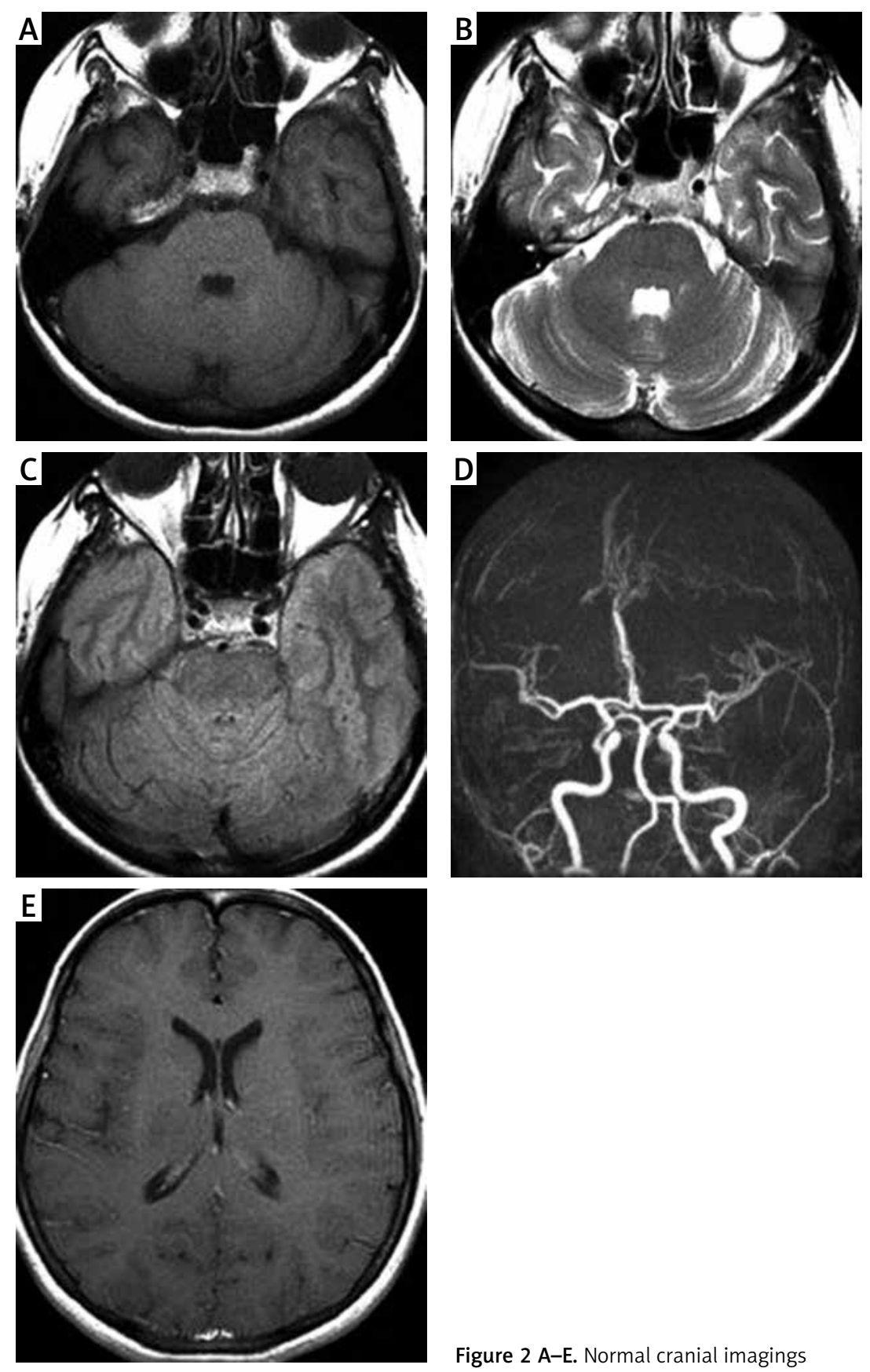

Figure 2 A-E. Normal cranial imagings 

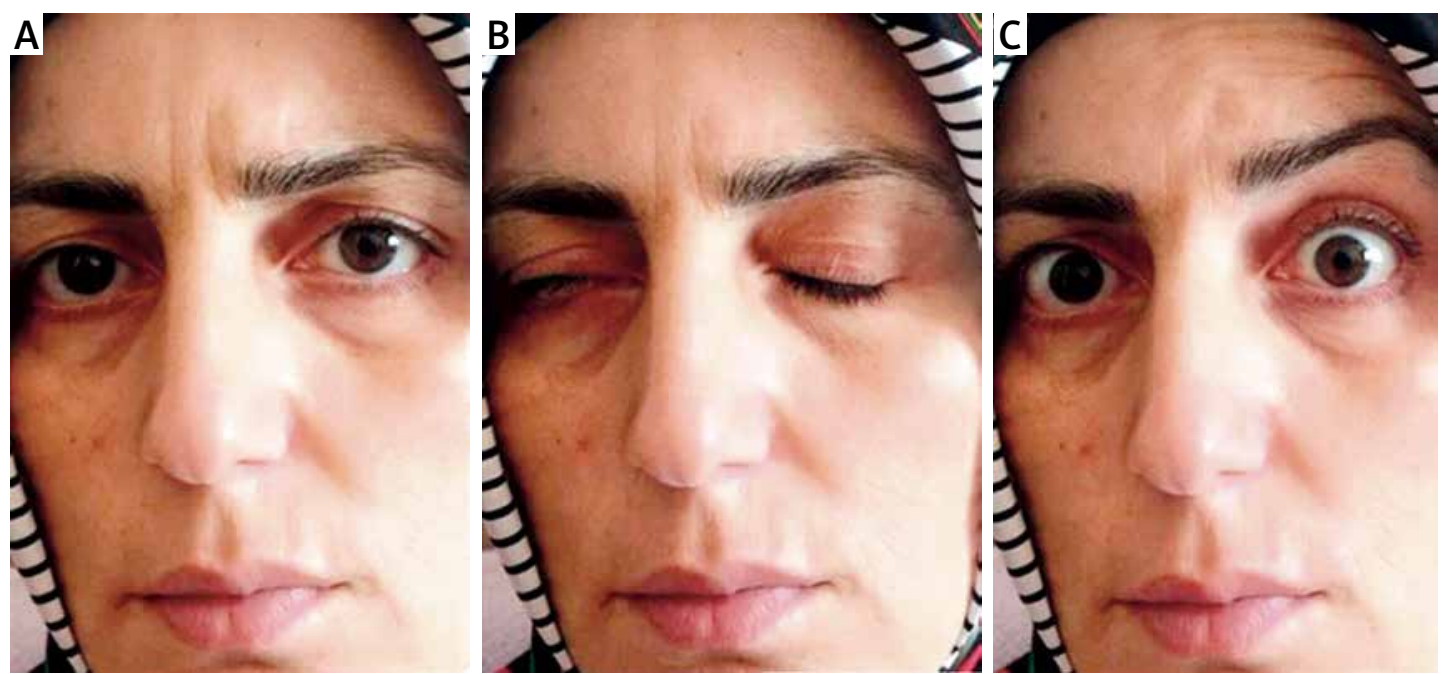

Figure 3 A-C. Right peripheral facial palsy and right abducens paralysis on neurologic examination
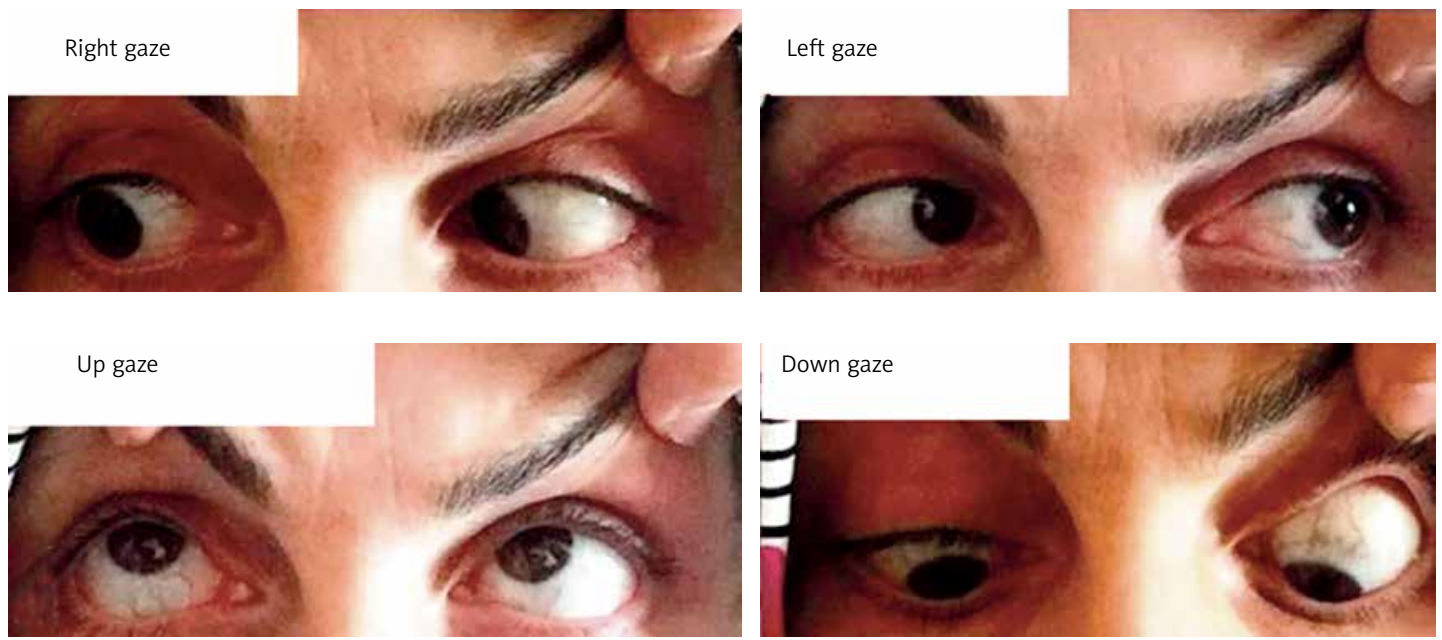

Figure 4. Control cranial imaging which was normal

Cranial MRI and MR diffusion, MR arteriography and venography studies were repeated, but there was no evidence of pathological imaging findings (Figure 4). Our case was evaluated as HaNDL syndrome, because the headache was concurrent with neurologic deficiency, other causes that may cause CSF pleocytosis were excluded, there was a clinic fluctuation during 3-month, and the symptoms had completely regressed at the end of the second month. Headaches were partially controlled with topiramate, and there was no recurrence of symptoms and complaints.

HaNDL syndrome should be carefully considered in a patient with headache, recurrent episodes of neurological disorders, lymphocytic pleocytosis in CSF and presence of normal imaging findings. HaNDL syndrome was classified in secondary headaches associated with an intracranial extravascular cause by an international society in 2004 [2, 4]. Headache of HaNDL syndrome is usually seen in severe throbbing-compressor style, bilateral or unilateral, and can last for an hour to a week. In our case the headache was of throbbing character and was resistant to medical treatment. In HaNDL syndrome, lymphocytic pleocytosis and elevated protein levels in CSF are often seen as laboratory findings. In our case, it drew attention to lymphocytosis in CSF, but protein levels were within normal limits. However, our patient was thought to have HaNDL syndrome with normal neurological scanning and exclusion of infection. Clinical symptoms of HaNDL syndrome always show full recovery and often have repetitive episodes. On follow-up of our case, the symptoms were nearly resolved and did not repeat at the end of the $8^{\text {th }}$ month.

Neurologic deficits in HaNDL syndrome may take the form of sensory, motor, visual, and speech impairment [3]. Rare symptoms of HaNDL syndrome are blurred vision, photophobia, homonymous hemianopia, cortical blindness, papilledema, increased intracranial pressure, and $6^{\text {th }}$ cranial nerve 
palsy and external ophtalmoplegia syndrome [4, 5]. If patients who have sixth cranial nerve involvement are examined, it is striking that increased intracranial pressure accompanies this syndrome. Our case showed unilateral cranial nerve involvement. However, our patient, a cranial nerve limited HaNDL syndrome case, is the first of its kind in the literature with V1 (right maxillary trigeminal nerve hypoesthesia in the sensory areas), VI (right eye inward gaze palsy), and VII (right peripheral facial paralysis) cranial nerve palsy and without increased intracranial pressure syndrome.

The patient provided written informed consent.

\section{Conflict of interest}

The authors declare no conflict of interest.

\section{References}

1. Swanson JW, Bartleson JD, Whisnant JP. A migrainous syndrome with CSF pleocytosis. Neurology 1980; 30: 418.

2. Headache Classification Subcommittee of the International Headache Society. The international classification of headache disorders. 2nd edn. Cephalalgia 2004; 24: 9-160.

3. Coban A, Shugaiv E, Tuzun E. Syndrome of headache accompanied with transient neurologic deficits and cerebrospinal fluid lymphocytosis. Arch Neuropsych 2013; 50: $52-5$

4. Morrison DG, Phuah HK, Reddy AT, Dure LS, Kline LB. Ophthalmologic involvement in the syndrome of headache, neurologic deficits, and cerebrospinal fluid lymphocytosis. Ophthalmology 2003; 110: 115-8.

5. Chan JW, Cheng C. Complete external ophthalmoplegia in headache, neurologic deficits, and cerebrospinal fluid lymphocytosis (HaNDL) syndrome. Eye Lond 2010; 24: 198-9. 